\title{
ANÁLISIS DE LA EXPRESIÓN DE LA CORTESÍA EN RTVE* INTERNACIONAL PARA LA ENSEÑANZA DEL ESPAÑOL-LENGUA EXTRANJERA**
}

\author{
ANALYSIS OF EXPRESSION OF POLITENESS OF INTERNATIONAL \\ RTVE FOR TEACHING SPANISH AS A FOREIGN LANGUAGE
}

\author{
NOELIA M. RAMOS-GONZÁLEZ \\ Università Degli Studi del Molise. Campobasso, Italia \\ noelia_ramos_go@hotmail.com \\ ANA M. RICO-MARTÍN \\ Universidad de Granada. Melilla, España \\ amrico@ugr.es
}

\section{RESUMEN}

El fenómeno de la cortesía como contenido sociocultural de la enseñanza de una lengua extranjera ha sido objeto de estudio de muchas investigaciones actuales bajo la justificación de que el desconocimiento de sus elementos y normas puede generar un choque cultural entre un aprendiz de una lengua extranjera y un nativo de la misma. En este trabajo se aborda el estudio descriptivo de este fenómeno a través de cinco series de RTVE emitidas por Internet con el objeto de ofrecer un recurso válido para la enseñanza del español como lengua extranjera (ELE). Se analiza de forma cualitativa la expresión de la cortesía mediante diferentes elementos lingüísticos, paralingüísticos, quinésicos, proxémicos y cronémicos de los actos de habla contenidos en la muestra seleccionada y, de forma cuantitativa, la presencia de estos elementos. Se concluye en la necesidad de trabajos pragmáticos parecidos con el fin de dotar al alumno de ELE de estrategias diferentes de las de su lengua materna que le permitan adecuarse a una situación comunicativa concreta.

Palabras clave: Cortesía, actos de habla, contenidos socioculturales, aprendizaje del español-lengua extranjera, teleseries, Internet.

\footnotetext{
* Siglas de Radiotelevisión Española, organismo estatal que gestiona el servicio público de la radio y la televisión española.

* El artículo se enmarca en la línea de investigación denominada "La investigación en Didáctica del español como L2 o LE" (Cod.703.99.254), vinculada al Programa de Doctorado de la Universidad de Granada "Didáctica de la Lengua y la Literatura" (Cód. 100.56.8).
} 


\begin{abstract}
The politeness principle as sociocultural content in the teaching of foreign languages has been the topic of many current research works, because the ignorance of its elements and rules can generate a culture shock between a trainee of a foreign language and a native speaker. This paper approaches the descriptive study of this phenomenon through five RTVE series broadcasted on the Internet in order to provide a valuable resource for the teaching of Spanish as a foreign language. We analyze qualitatively the expression of politeness through different linguistic, paralinguistic, kinesic, proxemic and chronemic elements of speech acts in the selected sample, and quantitatively the presence of these elements in the serials. We conclude on the need of pragmatic works similar to this type in order to provide Spanish foreign language students with different strategies from those in their mother tongue that allow them to fit a particular communicative situation.
\end{abstract}

Keywords: Politeness, speech acts, sociocultural contents, Spanish as a foreign language learning, TV serials, Internet.

Recibido: 21/06/2012. Aceptado: 30/01/2013.

\title{
1. INTRODUCCIÓN
}

T a literatura en torno al fenómeno de la cortesía, sobre todo en las últimas déca¿das, ha acumulado una serie de estudios y trabajos de investigación que intentan acotar, clasificar y relacionarla con otros aspectos desde diferentes perspectivas. Sin embargo, resulta todavía hoy un tema muy interesante para la enseñanza de las lenguas extranjeras y, a pesar de toda esa literatura existente, el enfoque que se da a la cortesía y su tratamiento en los libros de textos dedicados a la enseñanza del español son aún escasos y, en muchos casos, estereotipados.

Este trabajo supone un acercamiento a la cortesía desde el punto de vista didáctico, donde puede encuadrarse dentro de los contenidos socioculturales, y en el que la literatura parece reducirse un poco más, dejando al descubierto lagunas en algunos aspectos, sobre todo en lo que respecta al tratamiento didáctico y a la inexistencia de materiales auténticos para trabajar las estrategias corteses en el aula de español como lengua extranjera (ELE).

Por tanto, en esta investigación se enlaza el fenómeno de la cortesía con la didáctica pasando a través de los medios de comunicación de masas. En otras palabras, se ha realizado un estudio descriptivo de los elementos que intervienen en las normas y conductas de cortesía del español peninsular reflejados en las series de televisión de producción española emitidas en su totalidad por Internet y, por tanto, accesibles desde cualquier punto del mundo con conexión, lo que permite considerarlas un recurso para el alumnado extranjero que desconoce cómo en- 
frentarse a determinadas circunstancias o no sabe descodificar adecuadamente el mensaje del interlocutor provocando situaciones que pueden resultar inadecuadas o embarazosas; todo ello bajo el convencimiento de la necesidad de utilizar conversaciones reales donde los alumnos puedan identificar todo tipo de registros y rasgos en actos comunicativos que les permitan adaptar su idiolecto al contexto situacional, para lo que las series de televisión suponen un enorme apoyo didáctico.

En el caso de este estudio, se ha optado por analizar series españolas por partir de un corpus unitario (sin distintas variedades de español) visible por Internet, sin embargo, se podría haber tomado muestras de cualquier país hispanohablante cuya programación televisiva tuviera la misma difusión internacional; de hecho, estas series han sido trabajadas en Italia por una de las autoras con la intención de desarrollar la competencia intercultural de jóvenes aprendices de ELE en ese país. Si se trabaja con un grupo de alumnos cuya competencia en la lengua extranjera es limitada, conviene empezar por una variedad de español para, una vez alcanzado cierto dominio lingüístico, trabajar con las restantes estableciendo comparaciones entre ellas.

\subsection{La cortesía y su estudio}

Definir el concepto de cortesía implica hacer referencia, asimismo, a los términos respeto y civismo, pero en la actualidad la ambigüedad y subjetividad del término cortesía, así como la proliferación de teorías y contrateorías, pueden llevar a grandes confusiones a los menos versados en la materia, sobre todo a la hora de tratarla en el aula.

Además, estos conceptos por sí solos no explican ciertos fenómenos relacionados con el aprendizaje de lenguas extranjeras, ya que, al hablar de cortesía, un diccionario suele hacer referencia a la demostración o acto con que se manifiesta la atención, respeto o afecto que tiene alguien a otra persona (RAE, 2007), pero no en todas las lenguas esto se muestra de la misma manera. Por otro lado, respeto y civismo están relacionados con la educación, algo que puede variar de una persona a otra y que va más allá de la lengua o del aprendizaje de un idioma. Por ejemplo, el hecho de que dos jóvenes nativos de lengua española que vivan en la misma zona puedan elegir hablarle de tú o de usted a su profesor variará según el grado de civismo que quieran transmitir o el background de educación que posean. Esto lleva a entender la cortesía como una manifestación multicultural, ya que sus muestras pueden ser numerosas y variar mucho de una cultura a otra, al igual que el civismo.

En general, como señala Bravo (2004), "es necesario ubicar el estudio de la cortesía en un área de la pragmática que se ocupe específicamente de las funciones de los recursos comunicativos desde una perspectiva sociocultural" (8) para que el hablante de una cultura concreta pueda interpretar correctamente todos los 
mensajes dentro de una situación de interacción real. Esto se produce si dicho hablante conoce y puede compartir determinados contenidos socioculturales con su interlocutor sin que entre ellos se produzcan malentendidos o confusiones, es decir, cuando sus comportamientos comunicativos formen parte de unos hábitos comunicativos compartidos.

Las autoras del presente trabajo toman como punto de partida las siguientes palabras de Bravo (2005):

Se trata de una actividad comunicativa cuya finalidad propia es quedar bien con el otro y que responde a normas y a códigos sociales que se suponen en conocimiento de los hablantes. Este tipo de actividad en todos los contextos considera el beneficio del interlocutor. El efecto que esta actividad tiene en la interacción es interpersonalmente positivo (33).

La autora introduce con esta cita el concepto de cortesía comunicativa, en este sentido, en estas páginas no sólo se abordan contenidos lingüísticos sino el aspecto sociocultural en general, englobando comunicación verbal y no verbal, para estudiar cómo se pueden aplicar a la didáctica cotidiana de los profesores de lengua extranjera.

Por otra parte, son muy variados los enfoques de estudio de la cortesía, es obligado mencionar su análisis siguiendo la teoría de los actos del habla de Austin (1962) y Searle (1969) y, a partir de ellos, a Haverkate (2004) cuando clasifica los actos del discurso en una situación comunicativa como asertivos, directivos, expresivos, paralingüísticos y metapragmáticos; y a Albelda (2005), que los considera en un corpus de conversaciones coloquiales del español peninsular, dividiéndolos entre corteses, descorteses y anticorteses.

Dentro de esta variedad, este trabajo analiza la cortesía desde la perspectiva de la sociolingüística, distribuyendo las expresiones de este fenómeno en torno a:

a) Formas de tratamiento. Constituyen un sistema de significación en el que se refleja la interrelación entre la cortesía como norma social y el sistema lingüístico, puesto que la elección de una u otra forma de tratamiento para una determinada relación social queda establecida por una serie de condicionantes extralingüísticas como la edad, sexo, posición sociocultural y el grado de intimidad (Alba y Sánchez, 1980). Entre estas formas se encuentran el nombre propio y el hipocorístico, los pronombres tú y usted, términos genéricos como niño/a, nene/a..., apelativos y apodos, motes y sobrenombres, etc.

Según Soler-Espiauba (1996), para un alumno extranjero resulta muy difícil saber qué pronombres de cortesía utilizar, cuándo y con quién, dada la línea tan sutil que discurre entre el uso del tú y del usted. Por tanto, el problema con el que se enfrenta el profesor de español va más allá de la estricta pedagogía del 
ELE, pues se halla más bien en el terreno de la sociolingüística. Esta dificultad se acrecienta cuando los materiales de ELE trabajados en Europa abordan el tuteo-voseo de forma simplificada y, con frecuencia, sólo dentro de la sección de cultura. Por ello, resulta de especial interés en el ámbito de la cortesía identificar el uso de los sistemas de tratamiento en cada una de las variantes del español para, posteriormente, acercarlo a las clases de ELE.

b) Formas convencionalizadas de cortesía. Sánchez, Alba y Pinilla (2009) las clasifican en saludos, despedidas, presentaciones, felicitaciones, agradecimientos, cumplidos, pésames, peticiones, ruegos y disculpas; son todos ellos actos de habla presentes en todas las culturas pero con diferentes grados de expresión, lo que puede llevar a un alumno de español a malinterpretar algunos de ellos o a mostrarse poco expresivo cuando interviene en un contexto de comunicación real.

c) Estrategias de cortesía. El español cuenta con un repertorio variado de formas lingüísticas que se enmarcan en la relación de cortesía y que los hablantes utilizan en numerosas ocasiones, aunque a veces puedan resultar meras formas lingüísticas estereotipadas, como pueden ser los saludos, o modificadas según el uso social y cultural.

Entre estas estrategias destacan la atenuación, cuyo objetivo es suavizar el mensaje por respeto al oyente, por superstición o por convención social; el eufemismo, para evitar la palabra con que se designa algo molesto, inoportuno... sustituyéndola por otra expresión más agradable; y el disfemismo, para trasgredir el convencionalismo social desde la perspectiva lingüística mediante un tono irónico, burlesco o humorístico. Briz (2004) incluye también los filtros evaluadores, aplicados tanto en la emisión como en la recepción del mensaje, que permiten explicar la evaluación de la cortesía antes y durante el proceso interaccional, y el uso de las estrategias corteses en torno a la solidaridad entre los interlocutores, el fin personal de la interacción, la pertinencia de idiomas, la temática y la aceptación lingüística y social.

Además, Albelda (2005) y Barros (2011) señalan la cortesía valorizante para reforzar la imagen de los interlocutores de forma directa (mediante halagos, alabanzas, cumplidos, agradecimientos...) o indirecta, a través de colaboraciones con el tema (afirmaciones, proargumentos...).

d) Expresiones extralingüisticas de cortesía. Bravo (2004) sostiene que la cortesía está extremadamente ligada a la noción de contexto, por lo que deberá analizarse de forma explícita el componente extralingüístico. Los elementos no verbales tienen un papel comunicativo importantísimo en el contexto de la cortesía a pesar de que la tradición de los estudios sobre las lenguas los haya descuidado. Se habla, pues, de paralenguaje (calidad vocal y recursos fónicos), 
quinesia (movimientos del cuerpo, conductas táctiles...), proxemia (forma de concebir el espacio, distancia entre los interlocutores...), cronemia (interpretación del tiempo), características físicas (aspecto, olores, colores...), artefactos (perfume, ropa, gafas...) y factores del entorno que aportan significado al acto comunicativo (muebles, arquitectura, luz...).

En este trabajo se ha seguido el estudio de Haverkate (1994) para el análisis verbal completado con aspectos relacionados con la cortesía comunicativa preconizada por Bravo (2004).

\subsection{La subjetividad de la cortesía y el concepto de transposición didáctica en el proceso educativo}

Según los elementos vistos hasta el momento, el concepto de cortesía puede adolecer de cierta subjetividad y esto influirá en la forma de enseñanza del ELE. En este sentido, hay que considerar la importancia de la transposición didáctica (Chevallard, 1985) como el instrumento que permite realizar una comprensión reflexiva y una explicitación de intenciones del acto de enseñanza-aprendizaje, de manera que se posibilite la transmisión pedagógica de los conceptos teóricos señalados a través del diseño de unidades didácticas para el aula donde se realice una sucesiva transformación de saberes, desde el saber teórico hasta el saber del alumno.

En el caso concreto de la cortesía, el esfuerzo debe intensificarse aún más, ya que los diversos estudios sobre este fenómeno han sido enfocados fundamentalmente a la teoría y no a la práctica diaria. Además, el docente deberá luchar contra los efectos perversos de tal transposición, como son la sincretización, o tendencia a reducir los contenidos didácticos por falta de tiempo para profundizar en ciertos aspectos, y la desnaturalización, o reducción del trabajo en el aula al bricolaje de actividades sin coherencia con el proyecto educativo por seguir, a causa del desconocimiento de los últimos estudios sobre la cortesía.

El deseo de trabajar con un aspecto tan subjetivo como es el de la cortesía y a través de series de televisión, donde la interacción oral es el objeto de análisis, complica aún más la tarea del docente, a esto se le suma la intención de que los ejemplos sirvan de muestrario para la práctica diaria en el aula.

Para cerrar este estudio se incluyen más adelante unas líneas metodológicas básicas que orientan el tratamiento de la cortesía en el aula.

\subsection{La televisión como medio transmisor de cultura}

A la hora de elaborar actividades comunicativas para el aula de ELE existen 
algunas limitaciones que pueden deberse a la dificultad de satisfacer el mayor número de necesidades comunicativas que surgen en la vida cotidiana, con sus diferentes tipos de interacción y clases de lenguaje. No obstante, lo más parecido al lenguaje exterior de cada día se puede extraer de las series de televisión ya que de ellas se obtienen distintos acentos, velocidades en el discurso, diversos tipos de complejidad lingüística y nivel de formalidad, así como un amplio abanico de secuencias comunicativas que ayudan a compensar las limitaciones lingüísticas de los aprendices (Vivas, 2009).

Además, los diálogos aparecen contextualizados por la imagen (el escenario, la forma de actuar, de vestir y los gestos de los personajes ayudan a captar mejor su personalidad y facilitan la comprensión de lo que se escucha), cuyos elementos apoyan la interpretación de los lenguajes verbal y gestual, y la descodificación correcta de los mensajes en el momento de la interacción.

Por otro lado, la televisión como recurso didáctico es un elemento del espacio innovador en la práctica docente al posibilitar el análisis de estrategias cognitivas, procedimentales y actitudinales de los alumnos (Santibáñez, 2005) y desarrollar sus competencias pragmáticas (Sibón, 2005); de hecho, en palabras de Medrano, Cortés, Aierbe y Orejudo (2010), "será preciso desarrollar competencias acerca del discurso televisivo, su lenguaje, su tipología, su gramática y sus géneros" (19). Además, se trata de un vehículo eficaz de contenidos socioculturales que permiten vivir la historia dentro del aula. A este respecto, estos últimos autores señalan que hay que aprovechar el papel enculturizador de este medio por el que deviene una de las grandes influencias culturales en nuestra sociedad; siendo así, revela distintas formas de vida, de comportamientos y actitudes que lo convierten en una fuente de aprendizaje para los telespectadores de cualquier edad.

\section{METODOLOGÍA DE LA INVESTIGACIÓN}

El objetivo principal de esta investigación es comprobar si las series de RTVE en Internet poseen elementos de cortesía del español que puedan ser analizados y catalogados para su posterior uso en la clase de ELE, para lo que se realiza un análisis descriptivo y observacional (Hurtado, 2000) que permite clasificar y cuantificar esta tipología de ejemplos.

Para esto se ha seleccionado una muestra intencional de cinco series actuales de producción española con diferente temática, difundidas por Internet en su versión completa, para privilegiar las que muestran una España real y de aspectos socioculturales cercanos, eludiendo las de carácter histórico o fantástico. Además, Internet facilita que alumnos y profesores puedan acceder a ellas en cualquier momento y lugar con conexión. Constituían la muestra las series "Unidad Central Operativa”, "Herederos", "Los misterios de Laura", "Gran Reserva” y "Pelotas". 
Las variables consideradas en el estudio son aquellos rasgos-tipo que definen la cortesía en lengua española y que se distribuyen entre elementos lingüísticos (registros, formas convencionalizadas de cortesía y actos de habla expresivos, turnos de palabra, cortesía valorizante, estrategias de cortesía positiva y uso de atenuadores) y elementos de comunicación no verbal (CNV) (signos paralingüísticos, quinésicos, proxémicos y cronémicos).

Se emplea la técnica de observación estructurada (Anguera, 1989) para el estudio cualitativo de los patrones de comportamiento observados, recogidos en una ficha de registro de elaboración propia validada por un juicio de diez expertos en el tema y en análisis de la comunicación oral.

El procedimiento seguido en la investigación incluía el visionado de los cinco primeros capítulos de la primera temporada, eligiendo dos de cada una de las series. A partir de ahí, según el sistema de transcripción del Grupo Val.Es.Co (Briz, Hidalgo, Padilla, Pons, Ruiz, Sanmartín y Albelda, 2002), se transcriben las secuencias donde se ha encontrado contextualizada la cortesía y se analizan de forma cualitativa los elementos vinculados. Posteriormente, se realiza un análisis cuantitativo relacionando el tiempo de visionado con el destinado al fenómeno en cada capítulo para comprobar su frecuencia de aparición.

\section{ANÁLISIS Y RESULTADOS DE LA INVESTIGACIÓN}

\subsection{Análisis cualitativo}

Partiendo de autores como Haverkate (1994), se han contextualizado los actos de habla en las secuencias observadas, determinando tiempo, lugar, personajes y antecedentes de la conversación. Seguidamente, con los actos corteses y la estrategias indirectas vistas, se ha reconducido el análisis hacia el estudio de Bravo (2004) donde aboga por la cortesía comunicativa, más que la cortesía meramente verbal, realizando así un análisis sociopragmático de la misma.

En este sentido, se completa el modelo de Haverkate con las aportaciones de Albelda (2005), quien delimita la cortesía valorizante, y con la introducción de los atenuantes corteses en la conversación coloquial de Briz (1998a). Además, se ha empleado la clasificación de Sánchez et al. (2009) sobre las formas convencionalizadas de cortesía y el tipo de registro; y se revisan los parámetros individuados por Cestero (2004) para el análisis de la CNV.

\subsubsection{Elementos lingüisticos}

Entre los elementos lingüísticos como gran bloque de variables, el primero considerado es el registro formal o informal de los personajes. Cobra especial relevancia 
tanto el cambio verbal de segunda a tercera persona como el uso del pronombre personal (tú/usted). Además, dentro de la informalidad, se han revisado otras fórmulas de tratamiento que denotan mayor o menor cortesía como son los usos de:

- Nombres propios o hipocorísticos: en la serie "Pelotas" llaman Flo a Florencio; Bea a Beatriz; o Richi a Ricardo.

- Términos genéricos: en "Los misterios de Laura" la protagonista llama a sus hijos niños o gemelos. En "Pelotas" los personajes de Chechu y Bea, cuando hablan entre ellos, se refieren a Cris como la niña.

- Apelativos: en "Herederos", Antonio siempre llama a Rafael Maestro porque es un torero; y Verónica, Julia y Jacobo llaman a Teresa Tata porque es quien los ha criado. Este último es un apelativo cariñoso para designar a la empleada de hogar que cuida de los niños pequeños de la casa, de ahí esta voz "que probablemente tenga su origen en el balbuceo infantil” (Hervás, 2010: 721); en cambio, en Hispanoamérica ha existido desde hace mucho tiempo para referirse cariñosamente, por ejemplo, a un sacerdote muy apreciado (en México, en el siglo XV los indios llamaban Tata Vasco al obispo Vasco de Quiroga) o a un ser superior, sea divino (Tata Nsambi de las religiones afrocubanas), sea terrenal (Tatadiós en Guatemala); en estos tres ejemplos no existe la misma justificación articulatoria del caso infantil.

- Apodos, motes, sobrenombres: en "Unidad Central Operativa" el equipo llama al personaje representado por la actriz Pastora Vega la Cara Guapa y Blanca llama afectivamente a su novio Perroflauta ${ }^{1}$.

En cuanto al uso del pronombre túlusted, se encuentran, obviamente, muchos ejemplos en los capítulos de las series. En "Unidad Central Operativa”, sólo en el primer capítulo se observa cómo el protagonista, el capitán Sierra, utiliza continuamente el pronombre usted con las personas que trabajan fuera de su equipo, es decir, las personas a las que interroga por estar implicadas en un caso, normalmente este uso le sirve como estrategia para infundirles respeto y mantener las distancias.

Cap.1. Secuencia 24 (51:11- 51:30). Contextualización: Sierra interroga a la hija de la dueña del bar en el mentidero. Está muy nerviosa. Le está preguntando para que confiese dónde está el bebé de Sara:

\footnotetext{
${ }^{1}$ Según la Fundación del Español Urgente se utiliza este término "para referirse a un tipo de persona, habitualmente joven y con aspecto descuidado, que puede verse como un hippy en su acepción más moderna. Se les denomina así porque suelen llevar perros y tocar la flauta, aunque el término ha trascendido su significado original y se utiliza en muchas ocasiones de forma despectiva para referirse a cualquier joven con aspecto desaliñado" (recuperado de http://www.fundeu.es/recomendacion/ perroflauta-en-letra-redonda-y-enuna-sola-palabra-956/).
} 
Hija de la dueña del bar: Oiga... Pero ¿por qué me habla así? (Está muy asustada, no quiere mirarlo a los ojos). Madre, ¿dónde está mi madre?

Sierra: (Interrumpiéndola) ¿Usted cree que Sara habría arriesgado asi la vida de su hija?

Hija de la dueña del bar: No voy a hablar más si no está mi madre... (Sigue muy asustada, sigue sin mirarle).

Con el resto de su equipo, Sierra intenta que lo tuteen todo el tiempo:

Cap. 1. Secuencia 16 (29:23-29:36). Contextualización: Marina le entrega el informe a Sierra en la UCO:

Marina: Lo que me pidió de Anselmo.

Sierra: De tú, hija, de tú, que los años... los tengo igual.

Además es frecuente el empleo de Don/Doña para llamar a los patriarcas y a la matriarca de las dos sagas familiares de "Gran Reserva", igualmente es utilizado el término Señorita y el apellido en el primer capítulo. Asimismo, se han encontrado ejemplos considerados en desuso: Señorita, para llamar a las chicas del personal de servicio en "Herederos":

Cap. 2. Secuencia 20 (44: 45). Contextualización: Para evitar que la noticia del romance entre el torero Rafael y Julia salga a la luz, Carmen contrata a una chica para que haga de doble de Julia. Después invita a cenar al director de la cadena, a quien sorprende presentándosela:

Carmen: Señorita, pase, por favor...

Chica: Gracias, Señora... (Apareciendo delante del director de la cadena una chica que podría ser la doble de Julia por su gran parecido físico. El director se queda sin palabras, muy sorprendido).

Para analizar las formas convencionalizadas de cortesía, segunda variable lingüística, se ha seguido el esquema de Sánchez et al. (2009):

- Saludos, desde fórmulas más formales (Buenos días en "Gran Reserva”, entre otras) hasta las más informales (Buenas, Antúnez, ¿tú por aquí? en "Pelotas"); despedidas informales son: taluego [hasta luego], Richi. Taluego, Presi en "Pelotas" frente a la formal de "Unidad Central Operativa" cuando el personaje de Elena se despide en el segundo capítulo de la serie: Ahora, si me disculpan, me voy a retirar; presentaciones formales e informales, entre estas últimas tenemos, en una escena del capítulo primero de "Unidad Central Operativa" las de Segura, quien presenta al resto del equipo de guardias civiles al capitán Sierra, jefe de la Unidad; un puesto de trabajo tan jerarquizado por la Guardia Civil hace que Segura tenga que utilizar el grado que tiene cada uno dentro del grupo. 
Cap. 1. Secuencia 4 (08:20- 08:40).

Segura: Os presento al capitán Sierra. (Mientras Sierra se va acercando a ellos, el resto se va levantando y acercándose a él. Miradas de recelo y, por ejemplo, Pablo se mete los faldones de la camisa dentro del pantalón, mientras que Marina deja de mordisquear algo que tenía en la boca. Segura, con el brazo, va indicando a cada uno) La alférez Marina Izquierdo, el Cabo Pablo Molina.

- Sobre el agradecimiento es interesante señalar, en la interacción entre un camarero y su cliente, que este último no suele dar las gracias al camarero al servirle algo si esto es rutina con un cliente habitual, como ocurre en el capítulo cuarto de "Pelotas", cuando la acción transcurre en el bar de La Unión.

- El cumplido ha sido identificado con la cortesía valorizante directa y con el piropo típicamente español. En "Herederos" se pueden enumerar los piropos hacia Carmen Orozco en el ruedo: ;Guapa! y también los relacionados con el toreo a Rafael: ;A la jubilación por la puerta grande!, aludiendo a la expresión taurina salir por la puerta grande por la que se hace el reconocimiento final del torero en una tarde de faena.

- El pésame, del que sorprendentemente se han hallado ejemplos ya en los primeros capítulos de cada serie. Ejemplo de "Gran Reserva” serían las palabras de Vicente: Lo siento (dando la mano a Daniel). // Lo siento mucho, Sofía. Jesús era como un hermano para mí (a Sofía, después de darle un beso en la mejilla).

- La petición y el ruego; el tipo de petición encontrado dependía de la caracterización del personaje, pues, según Haverkate (1994), la realización cortés del acto del habla depende de la distancia, el poder y el grado de imposición de los interlocutores, por ejemplo, el personaje de Blanca con su padre en el capítulo segundo de "Unidad Central Operativa", quien le ruega que le deje acompañarle, sin utilizar la fórmula por favor, sino expresando su deseo (iPuedo ir contigo? Jo, papá. Es la oportunidad de estar un poco juntos), al igual que el uso del modo imperativo seguido de por favor o gracias, lo que las convierte en órdenes corteses (Doña Concha, venga conmigo, por favor, frase de un guardia civil para detener a una sospechosa en Unidad Central Operativa), como utilizar la primera persona del plural seguida de gracias, para reducir la imposición: Traigamos, traigamos, gracias, cuando, en la misma serie, Sierra ordena a Marina que traiga ella misma un documento. Otras peticiones seguían el esquema de la fórmula ¿Te importaría... o realizando una petición en forma de problema, a la que el interlocutor apelado aporta una posible solución en su calidad de agente racional capaz de resolverlo, como ocurre en "Los misterios de Laura", cuando esta protagonista pide: Me siento un poco deshidratada. Ismael, ¿me traes un vasito de agua?

- La disculpa se ve en varios episodios asociada a los actos atenuadores del desacuerdo (Albelda, 2005), que sirven para disminuir el disentimiento con el otro 
y hacerle quedar bien preservando su imagen. Las voces perdón o perdona son usadas en las series cuando se tropieza con alguien, se llama a la puerta antes de entrar, se piensa que molesta o se llega tarde. En "Los Misterios de Laura", la inspectora es bastante despistada y le suelen pasar cosas de las que luego tiene que pedir disculpas, como ocurre en esta escena del primer capítulo:

Cap. 1. Secuencia 11 (25:15-27:50). Contextualización: Laura va a la casa donde se cometió el delito. Entra en la habitación de Joaquín, el hermano de la persona asesinada. Está jugando al póker por Internet. Laura y Joaquín hablan de jugar en Internet y ella dice que se lo juega siempre en la primera carta, dándole tranquilamente a la tecla de apostar:

Joaquín: Esta es una página de verdad, señora, jacaba de joder 1500 euros!

Laura: ¡Ay! ¡Válgame Dios! Lo siento mucho, de verdad.

- En la expresión de la promesa y la invitación se han tenido en cuenta las condiciones previas, como habilidad, aceptabilidad y razonabilidad. Un claro ejemplo es la conversación entre Blanca y Sierra del mismo segundo capítulo de "Unidad Central Operativa", en el que ella le pide quedarse con él y Sierra se justifica diciendo que han hecho lo mejor para ella. Además, se ha señalado la condición esencial, característica de la expresión Te prometo que... o similares, así como la condición de sinceridad, en la que resulta primordial que el hablante tenga realmente la intención de llevar a cabo el acto prometido. Al igual que las expresiones vinculadas al acto de aceptar y rechazar una invitación, las de compromiso se realizan dando opciones al interlocutor para que pueda elegir entre varias o aquellas simbólicas como Está usted en su casa (en "Los Misterios de Laura" hacia la inspectora Lebrel).

Respecto a la tercera variable, los turnos de palabra, se ha considerado tanto el uso de estructuras simétricas como asimétricas del diálogo a través de las parejas adyacentes, así como el uso de las interrupciones en ámbitos con formalidad preestablecida; es el caso de la señora-sirvienta, con fórmulas como Perdóneme que le interrumpa, pero... utilizada frecuentemente por el ama de llaves en "Herederos". En otras ocasiones se produce un desequilibrio en los turnos de palabra: destacan dos personajes que condicionan la conversación constantemente, uno de ellos es Florentino ("Pelotas"), cuyas interrupciones se producen cuando está nervioso o cuando el tema de conversación resulta trascendental o complicado al hablar con el abogado o con su mujer; al tratarse en ambos casos de un contexto informal, suele considerarse habitual que el hablante español interrumpa cordialmente al interlocutor para aportar información, corroborar o incluso mostrar empatía con él. En este caso, aunque parezca carente de cordialidad, forma parte de las características del personaje. La otra protagonista es Carmen ("Herederos"), encarna 
al interlocutor que se complace en dejar callados al resto por su influencia en la familia y su fuerte carácter; de hecho, es quien organiza los turnos de palabra cada vez que se habla con ella.

La cuarta variable es la cortesía valorizante (Albelda, 2005), en este caso, indirecta, ya que la realizada directamente se ha visto a través del cumplido y el piropo. En "Pelotas" destaca una de las secuencias del quinto capítulo, en la que los amigos de Flo intentan convencerle de las buenas cualidades de Kim-ki y aportan argumentos colaboradores que les ayuden a corroborar su versión:

Cap. 5. Secuencia 5 (09:22-10:40).

Javi: Bueno, y aunque saliese... el chico es majo.

Don Antonio: Y formal.

Flo: $Y$ chino, ;Chino, chinoooo! Que sea majo y formal eso es opinable, pero que es chino... eso es objetivo... ¡No jodáis!

Siguiendo a Haverkate (1994), las siguientes variables lingüísticas son las estrategias de cortesía positiva: aquéllas realizadas para intentar salvaguardar la imagen positiva del interlocutor. Las categorías que analiza el autor pueden ser de tipo lingüístico o metalingüístico, en este plano incluye las de indole empática y las de persuasión coactiva: cuando se usan estrategias para atenuar una opinión divergente, en la que el hablante puede que acentúe con modestia fingiendo ignorancia o incompetencia, o cuando se usan construcciones adversativas Tienes razón pero... o con el sentido léxico positivo de bueno, ligándose a la función atenuadora. Entre las estrategias de indole empática, se han extraído ejemplos de la variante profiláctica (evitando temas controvertidos que puedan dañar la sensibilidad del interlocutor) y de la variante alterocéntrica (sacando a colación temas de interés personal introducidos preferentemente con preguntas sobre la salud, el trabajo o la familia del interlocutor). Por otra parte, en la cortesía positiva también hay ejemplos de repetición empática de las palabras del otro para mostrar solidaridad: durante una secuencia del capítulo quinto de "Pelotas", en una conversación entre el abogado de la Clínica donde murió Elena, Flo y Antúnez, cuando el segundo decide no aceptar el dinero de la indemnización y acude con Antúnez para manifestar su decisión:

Cap. 5. Secuencia 22 (30:30-32:41).

Flo: No puedo.

Abogado: ¿Cómo que no puede?

Flo: Pues, que no puedo.

Abogado: Ya entiendo... que debo subir la cifra.

Flo: ¡No, no!

Antúnez: No, no es eso. 
Flo: No es eso. Verá al principio era entre quedarme sin Elena y sin un duro o quedarme sin Elena y con 100.000 euros. Pero no... no es tan sencillo.

Igualmente encontramos la estrategia pseudoinclusiva, cuando los hablantes fingen que en la actualización de lo descrito participan colectivamente ellos mismos y sus interlocutores. Normalmente se produce cuando la relación social es asimétrica, es decir, que el hablante tiene autoridad y poder sobre el oyente, como ocurre en "Gran Reserva", cuando Lucía hace una entrevista para un importante periódico de Nueva York, el entrevistador utiliza la primera persona del plural para que sienta que es una conversación en la que los dos van a intervenir o participar. Sin embargo, lo que le pide el entrevistador es que le hable de ella y de su currículum.

También se hace uso de la referencia indirecta para mitigar una crítica dirigida al interlocutor, normalmente utilizando una construcción pasiva sin agente especificado, es decir, el hablante deja de referirse abiertamente al oyente, por lo que el reproche adquiere un carácter indirecto. De esta forma se dirige el Coronel en el capítulo 1 de "Unidad Central Operativa": Aqui, en la UCO se trabaja en equipo. (Moviendo la mano y, sobre todo, el dedo índice). $Y$ no hay sitio para lobos solitarios.

Por otro lado, se encuentra también la justificación exhortativa del acto del habla, al proporcionar al interlocutor una información que no se ha pedido, mostrándose así cortés y presentándose como un interlocutor dotado de razón y capaz de motivar sus actos, reforzando su propia imagen positiva (primer capítulo de "Pelotas", donde Flo justifica, sin tener que hacerlo, la llamada de teléfono que ha recibido).

Para Haverkate (1994) una de las consecuencias principales del sistema de las máximas de Grice (1975) es que puedan ser burladas. Estas desviaciones de las normas (implicaturas) suelen servir como estrategias de cortesía, tales como la mentira piadosa: del primer capítulo de "Pelotas" se extrae un divertido ejemplo para que la abuela no se entere de algo que hizo su hija antes de morir.

En este ámbito, otra variable lingüística es el uso de los atenuadores (Briz, 1998b) para suavizar el mensaje por respeto al oyente, como en otra divertida secuencia del capítulo cuarto de "Pelotas", cuando Flo habla con Kim-ki para averiguar si está saliendo con su hija y da un rodeo hasta encontrar la pregunta adecuada:

Capítulo 4. (52:20-54:21).

Flo: ¿Y las mujeres españolas? ¿eh?... (Ruido del lavacoches)

Kim-ki: ¿Perdón?

Flo: Que si las mujeres, que si te gustan las mujeres...

Kim-ki: Sí. 
Flo: Claro, pero ¿te gustan mucho?

Kim-ki: Bueno... sí.

Flo: Es normal, ¿eh? A mí me gustan más que a un tonto una tiza, es normal... digamos que te gustan las mujeres, en general. Pero... ¿y en particular? Si... jvamos! 'que si tienes novia, kim- ki!

En "Unidad Central Operativa" la atenuación se utiliza en los interrogatorios, con expresiones como ¿Le importaría... para que la persona se relaje e intente contar lo que sabe, utilizando la influencia estratégica del condicional o del imperfecto, para modificar la fuerza de los actos exhortativos. La elección de verbos como poder y querer suponen otra estrategia habitual, como en las frases de Sierra en "Unidad Central Operativa" para evitar una orden a través de la mitigación.

Existen otras estrategias relacionadas con la manipulación del contenido conceptual del predicado, entre ellas destacan los eufemismos, frecuentemente relacionados con la muerte, la escatología o el sexo, por ejemplo, cuando el equipo de fútbol habla de la homosexualidad de Peter en "Pelotas". Sin embargo, también se utiliza el disfemismo cuando Richi emplea la palabra marica siendo homosexual o, por ejemplo, el utilizado por el Sargento Gutiérrez en "Unidad Central Operativa" para referirse al físico de la sargento Andrún: ¡Cómo está el Cuerpo!, al igual que para eludir el tema de la muerte utiliza levantarse la tapa de los sesos. La lítote es otra categoría de cortesía mitigadora de selección del contenido: en "Herederos", cuando el periodista no sabe cómo decirle a Carmen cuál es el problema y utiliza No es fácil en vez de Es difícil.

Por último, la ironía es otra estrategia habitual para comunicar cortesía, sin embargo, se trata de las más difíciles, ya que implica una interpretación que depende totalmente del contexto situacional. En las series "Los misterios de Laura", "Pelotas" y "Unidad Central Operativa" pueden encontrarse diferentes ejemplos de ironía, siendo menos propensos "Herederos" y "Gran Reserva", tanto por las características de los personajes como por el argumento de los capítulos.

\subsubsection{Elementos extralingüisticos}

El segundo gran bloque de las variables analizadas lo constituyen los códigos no verbales. Siguiendo a Cestero (2004), se consideraron primero los signos paralingüísticos, sean el tono, el timbre, la cantidad, la intensidad o el tipo de voz determinando o matizando la información; por ejemplo, en la serie de televisión "Herederos" el tono de voz de la protagonista condiciona la respuesta cortés o descortés de los interlocutores.

En el caso de las interjecciones, algunas tienen función cortés como el uso de $¿ E h$ ? al final de la frase para expresar solidaridad con el grupo, lo que Bravo (2004) 
denomina cortesía de afiliación, o con función interaccional, cuando con su uso implica la confirmación de la recepción del mensaje y esta interjección se convierte en indicador fático (Rodríguez, 2009). Otras encontradas para expresar alegría y emoción muestran también cortesía, como en "Los Misterios de Laura", cuando la protagonista se muestra muy contenta al oír que con la persona que está delante de ella, quien podría ser bastante diferente a ella, tienen en común su serie preferida de televisión: Guau, no me lo puedo creer... También es mi serie favorita... jla he dejado grabando esta noche! (Comienzan a hablar de la telenovela... Laura está muy emocionada).

Haverkate (1994) destaca el silencio con función tanto cortés como descortés; en el primer caso, en "Gran Reserva", ante la violencia de Gustavo, Paloma calla y responde con monosílabos para evitar enfrentamientos. Sin embargo, el silencio debe ser evitado si se recurre a la estrategia de la comunión fática: en el análisis de las teleseries, la ruptura de este silencio se observa en el segundo capítulo de "Herederos", cuando Cecilia ocupa constantemente el silencio mientras lava la cabeza a una señora en la peluquería.

Por otro lado, los signos quinésicos vienen dados por los movimientos y las posturas corporales que comunican o matizan el sentido de los enunciados verbales, por ejemplo, abrazos y palmadas en la espalda para saludarse, en el primer capítulo de "Unidad Central Operativa", cuando se reencuentran Luis y Sierra; al igual que en el primer capítulo, también, de "Pelotas", cuando muere la mujer de Flo y en el entierro Chechu le pone el brazo sobre los hombros en señal de apoyo al marido; o el empleo del movimiento giratorio de la mano para despedirse, abriendo y cerrándola, como el que hace Blanca en "Unidad Central Operativa".

Los abrazos, los besos y el apretón de manos en los entierros comunican también cortesía, ya que muestran la solidaridad con la familia de las víctimas. En "Pelotas", "Unidad Central Operativa" o "Gran Reserva" se encuentran casos de este tipo.

Respecto a los signos proxémicos, vinculados al concepto de espacio, pueden variar según el grupo cultural o la persona y también dependen del grado de formalidad o informalidad de la situación: en la fiesta de Carmen en "Herederos" las personas guardan la distancia pertinente. Sin embargo, el hijo, Jacobo, organiza una "fiesta alternativa" en la cocina de la casa con distancias mucho más cortas.

Por último, los signos cronémicos evidencian la concepción del tiempo en una cultura determinada. Aquí tienen su lugar la puntualidad de los personajes; los actos relacionados con la cronemia social, es decir, la pertinencia del acto en sí, como en "Gran Reserva" cuando se excusa Miguel por presentarse en casa de Sofía un día después de la muerte de su marido; o la cronemia interactiva, relacionada con la duración de ciertos signos (un abrazo, una mirada...). 


\subsection{Análisis cuantitativo}

Para este análisis se consideran dos variables: el tiempo de emisión de cada capítulo y el tiempo de las secuencias seleccionadas con rasgos de cortesía. Así pues, consiste en descubrir la cantidad de elementos reflejada a través del tiempo y el intervalo en el que se produce el fenómeno.

El valor total de tiempo de visionado entre los diez capítulos analizados ha sido de 11:44:55 horas (véase Gráfico 1), de las que más de tres horas eran destinadas a la cortesía (3:22:36 horas), con un amplio repertorio de variables, siendo el valor medio de los capítulos del 28.74\% (véase Gráfico 2).

TOTAL 11:44:55

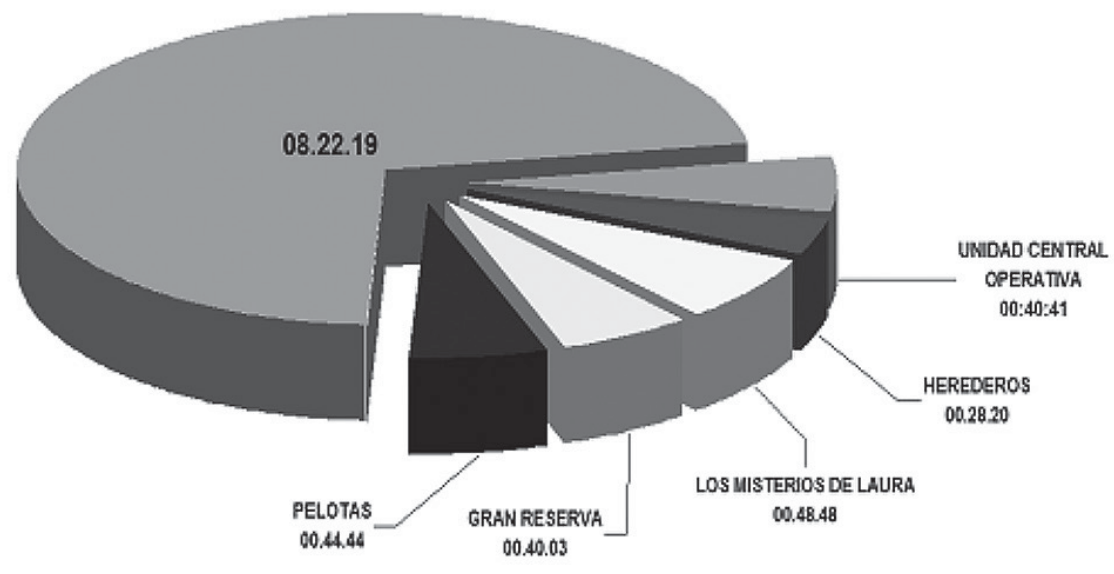

Gráfico 1. Tiempo visionado subdividido entre el dedicado a la cortesía y el restante.

Según el Gráfico 2, el porcentaje más alto está en "Los Misterios de Laura”, $30.72 \%$, mientras que "Herederos" evidencia el menor tiempo dedicado a la cortesía, 26.66\%, ya que, a pesar de la formalidad de los personajes, no utilizan tantas estrategias de cortesía, pues el autoritarismo de su protagonista conlleva frecuentes exhortaciones y frases carentes de cortesía. Por tanto, se puede apreciar que más del 25\% de cada una de las series puede ser seleccionado como ejemplo de variable de cortesía.

La semejanza de las columnas demuestra que el número de secuencias que contiene cortesía en cada serie es similar, ya que el argumento puede variar ligeramente el resultado, pero no de forma drástica. 


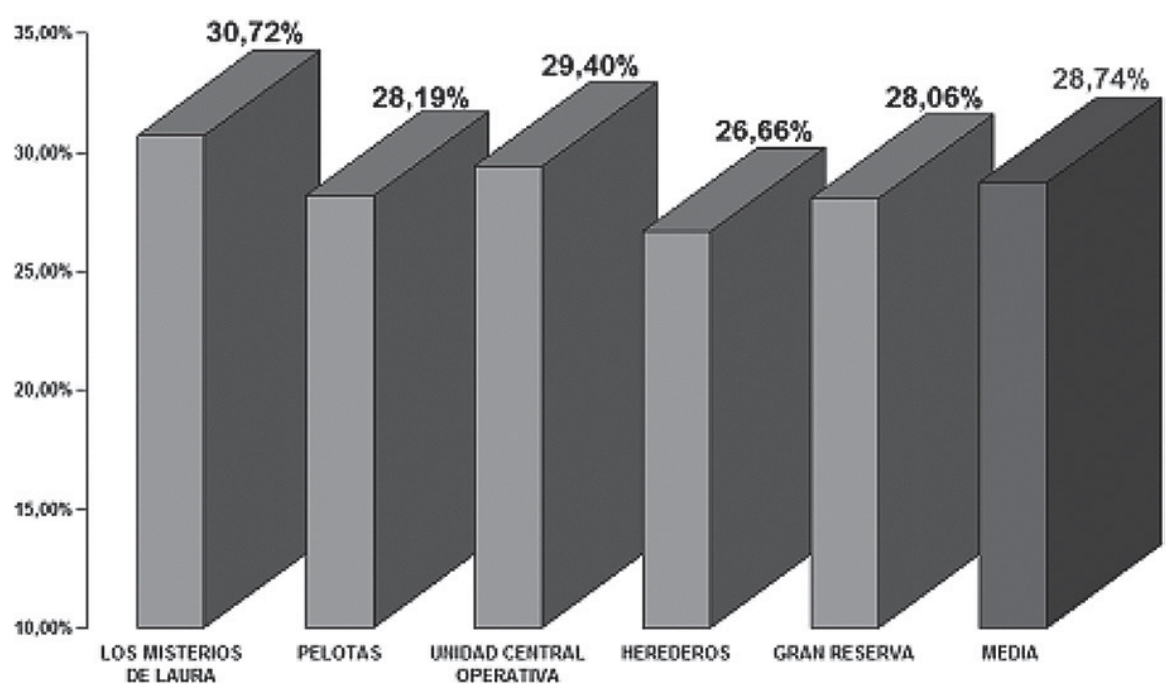

Gráfico 2. Porcentaje de cortesía en cada una de las series.

\section{DISCUSIÓN DE LOS RESULTADOS Y CONCLUSIONES DEL ESTUDIO}

A la luz de los análisis realizados, se puede afirmar la existencia de cortesía en todos los capítulos de las series de televisión. Además, ésta puede ser acotada, clasificada y, posteriormente, estudiada a través de la transcripción y contextualización de los diálogos.

El análisis cualitativo demuestra cómo las series no solo contienen elementos de cortesía, sino que revelan un amplio abanico de variables de cortesía actuales y contextualizadas, que pueden explicar por qué se produce el fenómeno, así como establecer categorías entre cada una de las variables como hacen Haverkate (1994), Calsamiglia y Tusón (1999) y Escandell (2004) con los actos corteses y la estrategia indirecta en situaciones contextualizadas. Por otro lado, en la línea de Bravo (2004), se ha realizado el análisis de la cortesía relacionado directamente con la comunicación, al considerarla un conjunto de conocimientos socioculturales útiles para interpretar de forma adecuada una situación, conocimientos que no deben ser asimilados sin más por el alumno de ELE, sino que debe interpretarlos $\mathrm{y}$ construirlos en una interacción social que puede llevarse a cabo con nativos $\mathrm{u}$ otros aprendices de ELE (Guillén, 2004).

Por otra parte, Ardila (2003) determina tres niveles de concreción de la cortesía, dos de ellos considerados también en este estudio: el cultural, es decir, característico de la cultura española, como es el uso de determinados saludos o el caso del camarero de un bar de una de las series, que no suele pedir por favor o dar 
las gracias porque los personajes son clientes habituales; y el idiosincrático, por ejemplo, cuando a la hora de saludar, presentarse o realizar el interrogatorio a los sospechosos en "Unidad Central Operativa”, los grados de cortesía vienen determinados por la personalidad del personaje.

Estas líneas llevan a destacar que las series de televisión son una verdadera muestra de diferentes tipos de cortesía. El más común, el referente al registro utilizado (formal o informal), cobra especial relevancia no sólo por los numerosos ejemplos de las series sino porque otros estudiosos también han dado muestras de su interés por éste (Albelda y Fernández, 2006).

Respecto a las formas de tratamiento, igual que en los trabajos de Alba y Sánchez (1980), Pedroviejo (2004) o Soler-Espiauba (1996), en las muestras analizadas queda patente el detrimento del usted a favor del tú, así como la tendencia a la desaparición del uso de las fórmulas de tratamiento nominales en el intercambio comunicativo y, por el contrario, el aumento del uso de las formas pronominales, con predominio del tú.

En el caso de las estrategias de cortesía, las series reflejan en gran parte de sus secuencias que el fin último de toda interacción es lograr la aceptación lingüística y social del otro, pues el hablante recurre a mecanismos de cortesía atenuadora para lograr metas difíciles, los factores que inciden en la presencia/ausencia de esta atenuación en conversaciones cotidianas se encuentran entre los recogidos por Briz (2005) en un corpus de entrevistas analizadas.

Acerca de la cortesía valorizante (Albelda, 2005; Barros, 2011), en esta investigación se analizan no sólo las directas e indirectas desde una perspectiva pragmalingüística, como hace la última autora, sino también sociopragmática.

Por último, otro objeto de estudio es la CNV, tema de gran amplitud y complejidad, caracterizado por su interdisciplinaridad, ya que comprende hábitos y costumbres culturales de una comunidad, incluida la cortesía, por lo que supone un verdadero apoyo para el componente sociocultural en la didáctica de ELE. Sin embargo, según Cestero (2004), necesitaría de un inventario de signos no verbales previo que facilite el aprendizaje, además de estudios comparativos interculturales, favoreciendo así la inclusión de la CNV en los diseños curriculares de enseñanza de lenguas extranjeras.

En cuanto al análisis cuantitativo, revela que la cortesía se presenta en todos los capítulos de las series, con una variedad media de expresiones del $28.74 \%$ que pueden ser observadas, cómo mínimo, un $26.66 \%$ del tiempo total del capítulo, cifras que dan relevancia al fenómeno, cuyo estudio resulta indispensable para el alumno de ELE con el fin de que no cometa errores pragmáticos, peor tolerados que los gramaticales.

Por otra parte, el hecho de utilizar las series de televisión como material para el análisis viene corroborado por Guillén (2004), quien apuesta por los mass media como instrumento para acercarse a la sociedad por la muestra que hacen de las 
prácticas sociales y de los intereses dominantes. Asimismo, se revelan didácticamente como materiales con un alto grado de disponibilidad, ya que, por ejemplo, las series analizadas se encuentran al alcance de cualquier profesor y estudiante que disponga de acceso a Internet estén donde estén. Además, sirven para estimular las destrezas heurísticas (de descubrimiento y análisis) pues los capítulos pueden ser parados, explicados, analizados, etc., tanto, por uno como por otro.

Incide en su idoneidad para trabajar la cortesía en clase el hecho de que, como apuestan Albelda y Fernández (2006), estas series incluyen conversaciones muy útiles para el estudio del registro coloquial. Aunque no completamente reales, son verosímiles pues las de producción nacional incluyen escenas que podrían ser cotidianas en una sociedad española como la actual. Asimismo, Baralo (2003) aboga por el uso de muestras auténticas que permitan al aprendiz reconocer diferentes variables en lo más parecido al lenguaje de cada día; en lo que respecta al tratamiento de cortesía, argumenta que no se trata de dominar todas esas variables sino de que sepa interpretarlas adecuadamente según la variante cultural en la que se encuentre.

Partiendo de estas premisas, se debe trabajar por el fomento de la interculturalidad en el aula para favorecer el conocimiento frente al preconcepto de culturas mejores o peores, de la misma forma que no existen éstas, tampoco existen culturas más corteses que otras. Cada una tiene su forma de pensar, sentir y actuar, y para entenderla se deben interpretar sus manifestaciones de acuerdo con sus propios criterios culturales, según Rodrigo (1999), evitando el estereotipo y los prejuicios. Se hace necesario un conocimiento más profundo de la cultura en cuestión que pasa por un estudio adecuado del uso de la cortesía española dentro del aula de ELE, teniendo en consideración que España y Latinoamérica son dos áreas geográficas con sus propias características lingüísticas y culturales, que también hay que transmitir a los alumnos que ya posean un cierto bagaje idiomático en esta lengua meta.

El paso posterior de esta investigación será utilizar las muestras analizadas para elaborar materiales didácticos útiles para la reflexión sobre la cortesía en las clases de ELE con el fin de que el alumno pueda adquirir las estrategias necesarias para adaptarse a cualquier circunstancia mediante un conocimiento sociocultural adecuado y, en consecuencia, una conciencia intercultural flexible que le permita adecuarse a cualquier contexto en la lengua que está aprendiendo. Ello implica diseñar un programa de intervención en el aula de ELE que favorezca la interpretación, primero, y el uso, después, de las normas de cortesía españolas como complemento al desarrollo del resto de las competencias comunicativas en esta lengua, de esta forma participarían como un elemento más en la enseñanza de la cultura para la formación integral del alumno, más allá de su enseñanza como información de datos sociopolíticos, geográficos y folklóricos diversos como se ha venido haciendo de manera tradicional (Areizaga, 2002). 


\section{ORIENTACIONES METODOLÓGICAS BÁSICAS PARA TRABAJAR LA CORTESÍA EN EL AULA DE ELE}

En el momento de llevar al aula el tratamiento de la cortesía, el primer paso es reconsiderar el papel tradicional del profesor de lenguas para transformarlo en un mediador intercultural, modelo de sus alumnos, conocedor de sus principales características culturales, con una amplitud de miras considerable y una tolerancia hacia los demás proveniente de una experiencia personal en multitud de situaciones comunicativas con interlocutores diversos. Ello le permitirá plantearse como objetivos didácticos: a) reconocer las diferentes dimensiones en las que se organizan las relaciones interpersonales en otras culturas, b) comprender mejor las formas de pensar, los valores y el mundo emocional de esas culturas, c) reconocer malentendidos y, en consecuencia, d) desenvolverse mejor en situaciones comunicativas como las trabajadas en el aula. En estos objetivos se aprecia la graduación de contenidos desde los meramente socioculturales (a), como son las normas de cortesía en ELE, hasta los más procedimentales (d), pasando por los de tipo afectivo (b y c).

Alcanzar estos objetivos requiere un enfoque metodológico muy flexible, de carácter colaborativo, como es el modelo interactivo-comunicativo a través de actividades de tipo dialógico, experienciales y de análisis personal sobre los elementos de cortesía percibidos en las series de televisión. El procedimiento de trabajo que se propone con un capítulo visionado sería: dentro del marco más amplio de un acto comunicativo, observación del hecho o conducta de cortesía que se pretende analizar; interpretación del mismo que cada alumno quiera aportar al resto; comparación con la cultura propia y propuesta de resolución de malentendidos y/o conflictos que pudieran darse, descartando la posible pertinacia etnocéntrica que puedan presentar algunos alumnos.

Hay que atender a todos los elementos propios de un acto de habla comunicativo que nos ofrecen las grabaciones audiovisuales que constituyen una teleserie: la intención de los interlocutores, la negociación y la interacción entre ellos, además de los elementos de la comunicación no verbal que pueden hacer efectivo o no ese acto de habla.

No obstante, existen diversos factores por considerar pues condicionan el éxito del modelo didáctico elegido: distancia entre las culturas que se relacionan, el contexto específico del alumnado, su origen, edad y formación, el contacto con la cultura hispana y, por último, el tiempo que puede dedicarse a la tarea de comprender la cortesía propia del ELE y de las culturas hispanas.

En lo que se refiere a la evaluación de estos contenidos de cortesía, no es difícil verificar las pautas y elementos de ELE que reconoce el alumno, pero sí lo es constatar el grado de integración de la cortesía que presenta al final del periodo de aprendizaje, dado que habría que observar sistemáticamente al aprendiz fuera 
del aula y en diferentes situaciones de comunicación con nativos hispanos para asegurarnos si existe tal integración.

Siguiendo el enfoque socioafectivo, otras orientaciones útiles para trabajar la competencia intercultural, donde se incluye el fenómeno de la cortesía, se encuentran en trabajos de Alonso (1998), Denis y Matas (2002), Rico (2005, 2009), Rico-Martín (2013) y Soler-Espiauba (2006), entre otros.

Estas han sido, pues, unas pautas básicas pero importantes para llevar al aula la cortesía en ELE. A partir del análisis realizado de sus elementos y de estas breves sugerencias, como se adelantó en el cierre del epígrafe 4, interesa, después, crear esos materiales didácticos que permitirán aprovechar las filmaciones para desarrollar estos contenidos específicos de la competencia intercultural, pero sin aislarlos de los restantes.

\section{REFERENCIAS}

Alba, V. y Sánchez, J. (1980). Tratamiento y juventud en la lengua hablada. Aspectos sociolingüísticos. Boletín de la Real Academia Española, LX, Cuadernos CCXIX, 95-130.

Albelda, M. (2005). El refuerzo de la imagen social en conversaciones coloquiales en español peninsular. La intensificación como categoría pragmática. En D. Bravo (coord.), Estudios de la (des)cortesía en español: categorías conceptuales y aplicaciones a corpora orales y escritos (pp. 93-118). Buenos Aires: Dunken.

Albelda, M. y Fernández, M.J. (2006). La enseñanza de los registros lingüísticos en E/LE. Una aplicación a la conversación coloquial. Marcoele. Revista de Didáctica, 3, 1-31. Disponible en http://marcoele.com. Consulta: 20.09.2013.

Alonso, R. (1998). Competencia comunicativa y cortesía. Cuestiones metodológicas. En R. Fente, A. Martínez y J. A. Molina (eds.), El español como lengua extranjera. Aspectos generales. Actas del I Congreso Nacional de ASELE (pp. 4352). Málaga: ASELE. Disponible en http://cvc.cervantes.es/ensenanza/biblioteca_ele/asele/pdf/01/01_0191.pdf. Consulta: 20.09.2013.

Anguera, M. (1989). Metodología de la observación en las ciencias humanas. Madrid: Cátedra.

Ardila, J.A.G. (2003). Variables sociopragmáticas de contextualización y niveles de concreción de la cortesía en castellano e inglés. Forum of Modern Language Studies, 39, 1-21. Disponible en http://vbn.aau.dk/files/62801165/SyD4_ardilla.pdf. Consulta: 20.09.2013.

Areizaga, E. (2002). El componente cultural en la enseñanza de lenguas: elementos para el análisis y la evaluación del material didáctico. Cultura y Educación, $14(2), 161-175$.

Austin, J.L. (1962). How to do things with words. Oxford: Oxford Clarendon Press. 
Baralo, M. (2003). Mestizaje e interculturalidad en la variación diatópica y su incidencia en español/LE. En M. Pérez y J. Coloma (eds.), El español, lengua del mestizaje y la interculturalidad. Actas del XIII Congreso Internacional de ASELE (pp. 152-164). Madrid: ASELE. Disponible en http://www.educacion.gob.es. Consulta: 20.09.2013.

Barros, M.J. (2011). La cortesía valorizadora en la conversación coloquial española: Estudio pragmalingüístico. Tesis doctoral. Universidad de Granada. Disponible en http://digibug.ugr.es/bitstream/10481/17612/1/19835851.pdf. Consulta: 20.09.2013.

Bravo, D. (2004). Panorámica breve acerca del marco teórico y metodológico. En D. Bravo (coord.), Pragmática sociocultural: estudios sobre el discurso de cortesía en español (pp. 5-11). Barcelona: Ariel Lingüística.

Bravo, D. (2005). Categorías, tipologías y aplicaciones. Hacia una redefinición de la cortesía comunicativa. En D. Bravo (coord.), Estudios de la (des)cortesía en español: categorías conceptuales y aplicaciones a corpora orales y escritos (pp. 2152). Buenos Aires: Dunken.

Briz, A. (1998a). El español coloquial en la conversación. Barcelona: Ariel.

Briz, A. (1998b). La atenuación en la conversación coloquial. Una categoría pragmática. En L. Cortés (coord.), El español coloquial. Actas del I Simposio sobre análisis del discurso oral (pp. 103-122). Almería: Universidad de Almería.

Briz, A. (2004). Aportaciones del análisis del discurso oral. En J. Sánchez e I. Santos (coords.), Vademécum para la formación de profesores: enseñar español como segunda lengua (L2), lengua extranjera (LE) (pp. 219-241). Madrid: SGEL.

Briz, A. (2005). Eficacia, imagen social e imagen de cortesía. En D. Bravo (coord.). Estudios de la (des)cortesía en español: categorías conceptuales y aplicaciones a corpora orales y escritos (pp. 53-91). Buenos Aires: Dunken

Briz, A., Hidalgo, A., Padilla, X., Pons, S., Ruiz, L., Sanmartín, J. y Albelda, M. (2002). La transcripción de la lengua hablada: El sistema del Grupo Val.Es.Co. Español Actual, 77-78, 57-85.

Calsamiglia, H. y Tusón A. (1999). Las cosas del decir. Manual de análisis del discurso. Barcelona: Ariel.

Cestero, A.M. (2004). La comunicación no verbal. En J. Sánchez e I. Santos (coords.), Vademécum para la formación de profesores: enseñar español como segunda lengua (L2), lengua extranjera (LE) (pp. 593-616). Madrid: SGEL.

Chevallard, Y. (1985). La transposition didactique; du savoir savant au savoir enseigné. Paris: La Pensée Sauvage.

Denis, M. y Matas, M. (2002). Entrecruzar culturas. Competencia intercultural y estrategias didácticas. Bruselas: De Boeck \& Larcier.

Escandell, M.V. (2004). Aportaciones de la pragmática. En J. Sánchez e I. Santos (coords.). Vademécum para la formación de profesores: enseñar español como segunda lengua (L2), lengua extranjera (LE) (pp. 179-197). Madrid: SGEL. 
Grice, H.P. (1975). Logic and Conversation. In P. Cole y J.L. Morgan (eds.), Speech Acts (pp. 41-58). New York: Academic Press.

Guillén, C. (2004). Los contenidos culturales. En J. Sánchez e I. Santos (coords.), Vademécum para la formación de profesores: enseñar español como segunda lengua (L2), lengua extranjera (LE) (pp. 835-851). Madrid: SGEL.

Haverkate, H. (1994). La cortesía verbal. Estudio pragmalingüistico. Madrid: Gredos.

Haverkate, H. (2004). El análisis de la cortesía comunicativa: categorización pragmalingüística de la cultura española. En D. Bravo (coord.). Pragmática sociocultural: estudios sobre el discurso de cortesía en español (pp. 55-65). Barcelona: Ariel Lingüística.

Hervás, Gl. (2010). La sociedad española en su literatura. Selección y análisis de textos de los siglos XVIII, XIX y XX. Madrid: Editorial Complutense.

Hurtado, J. (2000). Metodología de la investigación holística. Caracas: Sypal.

Medrano, C., Cortés, P.A., Aierbe, A. y Orejudo, S. (2010). Los programas y características de los personajes preferidos en el visionado de la televisión: diferencias evolutivas y de sexo. Cultura y Educación, 22(1), 3-20.

Pedroviejo, J.M. (2004). Formas de tratamiento en dos obras de teatro del siglo XX: "Historia de una escalera" y "Bajarse al moro". En D. Bravo (coord.). Pragmática sociocultural: estudios sobre el discurso de cortesía en español (pp. 245262). Barcelona: Ariel Lingüística.

RAE (Real Academia Española) (2007). Diccionario de la Lengua Española. 22a edición. Madrid: Espasa-Calpe.

Rico, A.M. (2005). De la competencia intercultural en la adquisición de una segunda lengua o lengua extranjera: conceptos, metodología y revisión de métodos. Porta Linguarum, 3, 79-94.

Rico, A.M. (2009). El profesor de ELE como agente hacia la transculturalidad. En A. Barrientos, J.C. Martín, V. Delgado y M.I. Fernández (eds.), El profesor de español LE/L2. Actas del XIX Congreso Internacional de ASELE. Vol. 2 (pp. 721-737). Cáceres: Universidad de Extremadura.

Rico-Martín, A.M. (2013). La competencia transcultural en el aprendizaje de una lengua no materna: desarrollo de actitudes positivas hacia la alteridad. Lenguaje $y$ Textos, 37, 171-182.

Rodrigo, M. (1999). La comunicación intercultural. Barcelona: Antropos.

Rodríguez, F.J. (2009). Estudio sobre las funciones pragmadiscursivas de ¿no? y ¿eh? en el español hablado. RLA Revista de Lingüistica Teórica y Aplicada, 47(1), 83-101.

Sánchez, J., Alba, V. y Pinilla, R. (2009). Aspectos del Español actual: Descripción, enseñanza y aprendizaje (L1 y L2). Acercamiento a la cortesía verbal, la creación neológica y la enseñanza del español. Madrid: SGEL. 
Santibáñez, J. (2005). Televisión: Un recurso para adquirir conocimientos, procedimientos y valores. Comunicar, 25, 363-364.

Searle, J.R. (1969). Speech Acts. Cambridge, Mass.: Cambridge University Press.

Sibón, T.G. (2005). La televisión, un medio en el aula. Comunicar, 25, 364.

Soler-Espiauba, D. (1996). ¿Tú o usted? ¿Cuándo y por qué? Descodificación al uso del estudiante de Español como Lengua Extranjera. En S. Montesa y P. Gomis (eds.), Tendencias actuales en la enseñanza del español como lengua extranjera I. Actas del V Congreso Internacional de ASELE (pp. 199-208). Málaga: ASELE.

Soler-Espiauba, D. (2006). Contenidos culturales en la enseñanza del español como 2/L. Madrid: Arco/Libros.

Vivas, J. (2009). El cortometraje como recurso didáctico en niveles iniciales (A1A2) de LE/L2. redELE, 17, 1-19. Disponible en http://www.educacion.gob.es. Consulta: 20.09.2013. 